\title{
Relationship Between Patellofemoral Finite Helical Axis and Femoral Trans-Epicondyle Axis Using a Static Magnetic Resonance-Based Methodology
}

\section{Zhenguo Yu}

Peking University Third Hospital

Hong Cai

Peking University Third Hospital

Bin Yang ( $\square$ yangbin@pkuih.edu.cn )

Peking University International Hospital https://orcid.org/0000-0001-7231-6678

Jie Yao

Beihang University

Ke Zhang

Peking University Third Hospital

Hua Tian

Peking University Third Hospital

Zhongjun Liu

Peking University Third Hospital

Research article

Keywords: patellofemoral joint, finite helical axis, trans-epicondyle axis, knee

Posted Date: December 29th, 2020

DOl: https://doi.org/10.21203/rs.3.rs-135789/v1

License: (9) This work is licensed under a Creative Commons Attribution 4.0 International License. Read Full License

Version of Record: A version of this preprint was published on March 24th, 2021. See the published version at https://doi.org/10.1186/s13018-021-02328-2. 
1 Title: Relationship between patellofemoral finite helical axis and femoral trans-

2 epicondyle axis using a static magnetic resonance-based methodology

3

4 Author name: Zhenguo $\mathrm{Yu}^{1}, \mathrm{MD}$, Hong Cai ${ }^{1}, \mathrm{MD}$, Bin $\mathrm{Yang}^{2}, \mathrm{MD} \& \mathrm{PhD}$, Jie Yao ${ }^{3}$, $5 \quad$ PhD, Ke Zhang ${ }^{1}, \mathrm{MB}$, Hua Tian ${ }^{1}, \mathrm{MD}$, Zhongjun Liu ${ }^{1}$, MD

7 Address 1: Department of Orthopedics, Peking University Third Hospital, No.49 North

8 Garden Road, Haidian District, Beijing 100191, China

9 Address 2: Department of Orthopedics, Peking University International Hospital, Life

Park 1, Zhongguancun Life Science Park, Changping District, Beijing 102206, China

Address 3: Key Laboratory for Biomechanics and Mechanobiology of Ministry of

Education, School of Biological Science and Medical Engineering, Beijing Advanced

Road, Haidian District, Beijing 100191, China

\section{E-mail address:}

Zhenguo Yu: yzguo3634@163.com

Hong Cai: hongcai@bjmu.edu.cn

Bin Yang: yangbin@pkuih.edu.cn

Jie Yao: yaojie@buaa.edu.cn

Ke Zhang: ZHANGKE@pkuih.edu.cn

Hua Tian: tianhua@bjmu.edu.cn 
0

1

3

4

Zhongjun Liu:puthztlzj@163.com

Corresponding authors: Hong Cai, Bin Yang, Jie Yao

Hong Cai:

E-mail: hongcai@bjmu.edu.cn

Fax: 010-82265201

Tel: 86-13501226970

Bin Yang:

E-mail: yangbin@pkuih.edu.cn

Tel: 86-13810803698

Jie Yao:

E-mail: yaojie@buaa.edu.cn

Tel: $86-13811034632$ 


\section{Declarations:}

\section{Ethics approval and consent to participate}

The protocol for the study project has been approved by the Ethics Committee of Peking University International Hospital (Approval number: YJ2017-020). All subjects received an oral and written explanation of the study and signed the informed consent.

\section{Consent for publication}

Not applicable.

\section{Availability of data and materials}

The datasets used and/or analysed during the current study are available from the corresponding author on reasonable request.

\section{Competing interests}

The authors declare that they have no competing interests.

\section{Funding}

This study was supported by grants from the National Natural Science Foundation of China (NSFC 11502014, 11302248, and 11421202), Young Elite Scientist Sponsorship Program by CAST (YESS 2015QNRC001), Peking University International Hospital Research Fund (YN2019ZD05), Open Project of Key Laboratory of Modern Measurement and Control Technology (Ministry of Education) (KF20181123206). 
Authors' contributions

69

(1) conception and design: BY, JY, HC and ZY; (2) algorithm establishment: JY; (3) imaging reconstruction and data analysis: ZY; (4) manuscript writing and final approval of manuscript: all authors. All authors read and approved the final manuscript.

\section{Acknowledgements}

We thank Dr. Xing Xin and Dr. Xingliang Wang for their assistance in the establishment of femoral trans-epicondyle axis. 


\section{Abstract}

Background: To manage patellofemoral joint disorders, a complete understanding of the in vivo patellofemoral kinematics is critical. However, as one of the parameters of joint kinematics, the location and orientation of patellofemoral finite helical axis (FHA) remains unclear. The purpose of this study is to quantify the location and orientation of the patellar FHA both in vivo and non-invasively at various flexion angles and to relate the FHA to the trans-epicondyle axis (TEA).

Methods: The Magnetic resonance (MR) images of 18 unilateral knees were collected at full extension and at $30^{\circ}, 60^{\circ}, 90^{\circ}$, and maximum angle of knee flexion. Threedimensional models of knee joint at different flexion angles were developed with the MR images, and were used to calculate the patellar tracking and FHA with a spline interpolation algorithm. By using a coordinate system based on the TEA, the FHA tracking was quantified. Six parameters concerning the location and orientation of the patellar FHA were analyzed.

Results: The average patellar FHA of 18 knees drew an L-shaped tracking on the midsagittal plane moving from the posteroinferior side of the TEA to the anterosuperior with knee flexion. Before $90^{\circ}$ flexion, the patellar rotational radius decreased slightly, with an average value of $5.65 \pm 1.09 \mathrm{~cm}$. During $20^{\circ}$ to $90^{\circ}$ knee flexion, the average angle between the patellar FHA and TEA was approximately $10^{\circ}$ and that between the FHA and coronal plane was maintained at about $0^{\circ}$, while that between the FHA and 
level plane fluctuated between $-10^{\circ}$ and $10^{\circ}$.

Conclusions: Patellar FHA was not fixed during flexion, which showed a close relationship with femoral TEA in both location and orientation. The results could help us better understand the patellofemoral joint kinematics and further deal with troublesome patellofemoral disorders.

Key Words: patellofemoral joint, finite helical axis, trans-epicondyle axis, knee

\section{Introduction}

Patellar maltracking is a common concern for managing disorders of the patellofemoral joint with high morbidity $[1,2]$. Because of many causes and subtle clinical features, patellar maltracking can be challenging to diagnose [3]. If focus merely on patellar tracking, there could be some inevitable disadvantages. High dependence on the coordinate system of patellar tracking has made the comparison among research results difficult, which led to the ambiguity to consensus of normal patellar tracking [4, 5]. To deal with patellofemoral disorders, it is crucial to fully understand the in vivo patellofemoral kinematics.

As one of the parameters of joint kinematics, the finite helical axis (FHA) of rotation has been used to describe joint motion for decades, especially in describing the motion of tibiofemoral joints [6] and ankle joints [7]. Unlike patellar tracking, the FHA is 
independent of the established coordinate system embedded in the "moving body" which can be directly applied to the analysis of joint forces and moments [8]. In the tibiofemoral joint, a close relationship between the FHA and femoral trans-epicondyle axis (TEA) was demonstrated [8]. There is relatively inadequate attention in relation with the patellofemoral FHA, however, in consideration for the coupled motion of the tibiofemoral and patellofemoral articulations, a similar relationship between patellar FHA and TEA might exist. Using a kinematics rig, Iranpour et al. [3] stated that the patella moved in a circle around the trochlear axis almost parallel to the femoral epicondylar and condylar axes. Another study by Coughlin et al. [9] showed that the patellar motion followed by a nearly perfect circular arc in the midsagittal plane of the femur, with an average best-fit radius of $30.6 \mathrm{~mm}$ during $0^{\circ}$ to $90^{\circ} \mathrm{knee}$ flexion. They also calculated the origin of this arc, that is the average patellar FHA, at $9.6 \mathrm{~mm}$ anterior and $11.6 \mathrm{~mm}$ proximal to the femoral TEA [9]. Unfortunately, the previous studies used invasive measurement techniques with in vitro knee, which might differ from the actual joint kinematics under physiological conditions.

Of particular importance, there were no descriptions about the location and orientation of patellar FHA at various knee angles, which was typically displayed as a series of straight lines encompassed by arcuate patellar tracking. Moreover, although the studies above measured the patellofemoral kinematics dynamically with optical trackers or position sensors [3, 9], quantitative results about the patellar FHA are still lacking. Thus, the primary purpose of this study was to quantify the patellar FHA and relate the FHA to the femoral TEA with a non-invasive and in vivo methodology based 
on static magnetic resonance (MR), whose accuracy to reconstruct continuous patellar motion has been corroborated [10].

\section{Subjects and methods}

\section{Subjects}

Eighteen healthy subjects participated in this study (Table 1). The study was approved by the Ethics Committee of Peking University International Hospital. All subjects received an oral and written explanation of the study and signed the informed consent form. Neither subjects had history of knee problems or pain, nor had clinically diagnosed knee pathology, previous knee joint surgery or contraindications to MR scan.

Table 1 Demographic characteristics of the subjects

\begin{tabular}{ll}
\hline Characteristics & Value \\
\hline Gender (male/female) & $9 / 9$ \\
Age $^{\mathrm{a}}$ (years) & $26.6 \pm 4.9$ \\
Height $^{\mathrm{a}}(\mathrm{cm})$ & $170.0 \pm 6.7$ \\
Weight $^{\mathrm{a}}(\mathrm{kg})$ & $61.1 \pm 9.2$ \\
$\mathrm{BMI}^{\mathrm{a}}\left(\mathrm{kg} / \mathrm{m}^{2}\right)$ & $21.1 \pm 2.5$ \\
\hline
\end{tabular}

${ }^{\text {a }}$ The values are given as the mean and the standard deviation.

\section{MR scanning}

The unilateral knee of each subject was scanned with the MR machine (Siemens/Verio 3.0T, Germany) at full extension and at $30^{\circ}, 60^{\circ}, 90^{\circ}$, and maximum angle of knee 
flexion. To accommodate the flexed knee in the magnetic field, subjects were positioned laterally. A thermoplastic knee fixator was designed to keep the target knee at the required angle and immobilized during scanning. The other leg was remained no contact with the target knee to avoid artifacts in the MR image. The following scanning parameters were used: fat-suppression T2-weighted image; slice increment $=0.999 \mathrm{~mm}$; slice thickness $=1 \mathrm{~mm}$; resolution $=512 \mathrm{pxl} \times 512 \mathrm{pxl}$; pixel size $=0.352 \mathrm{~mm}$. The MR images of the knee's sagittal section at five angles of knee flexion are shown in Fig. 1A.

\section{Geometry reconstruction and register}

Based on the MR images, three-dimensional (3D) models of the femur, patella, and tibia were developed by using the medical image processing software Mimics (version 16.0 Materialise, Inc., Belgium) (Fig. 1B). By applying the inverse engineering software, Rapidform (version 2006, 3D Systems, Inc., Korea), placing the femur models at various flexion angles in a fixed position, the patella and tibia of each position were registered to the femur models (Fig. 1C). The knee flexion angles were recalculated with reconstructed models. The process of knee flexion was divided into three stages: early stage $\left(0^{\circ}-45^{\circ}\right)$, middle stage $\left(45^{\circ}-90^{\circ}\right)$, and late stage $\left(>90^{\circ}\right)$.

\section{Calculation of patellar tracking and FHA}

Continuous patellar tracking was calculated with the above knee models at five angles of knee flexion. The motion process is shown in Additional file 1. The calculation method was developed and validated in our previous studies [10, 11]. In brief, m non- 
collinear reference points of the patella were determined $(\mathrm{m} \geq 3)$. The tracking of the reference points were derived from their coordinates at five angles of knee flexion with the order-three spline algorithm. Let radius vector $\mathrm{P}_{\mathrm{i}}(\theta)$ denotes the tracking of the reference points (for $\mathrm{i}=1,2, \ldots, \mathrm{m}$ ), and $\theta$ is the knee flexion angle. The patellar motion is characterised by an orthogonal rotation matrix $\mathrm{R}(\theta)$, and a translation vector $\mathrm{v}(\theta)$. The Lagrangian function $\mathrm{f}$ was defined as:

$$
\begin{gathered}
f(R(\theta), v(\theta))=\frac{1}{n} \sum_{i=1}^{m}\left[R(\theta) * P_{i}(0)+v(\theta)-P_{i}(\theta)\right]^{T} *\left[R(\theta) * P_{i}(0)+v(\theta)-\right. \\
\left.P_{i}(\theta)\right] \quad(1)
\end{gathered}
$$

The Lagrangian multiplier theorem was used to determine the $\mathrm{R}(\theta)$ and $\mathrm{v}(\theta)$ that minimise $f$ under the constraint condition of rigid motion:

$$
R^{T}(\theta) * \quad R(\theta)=\mathrm{I}
$$

where I is the identity matrix [12]. The accuracy of the calculated patellar tracking was validated with the precise tracking which was obtained from the motion capture experiment in our previous in vitro study[10].

Then, the FHA of the patellar motion was derived from patellar tracking with a $1^{\circ}$ increment of knee flexion angle. Each FHA is represented as a function:

$$
Y(\theta)=s(\theta)+x * n(\theta)
$$

where $\mathrm{n}$ is the unit vector along the FHA, and $\mathrm{s}$ is the radius vector of a point on the FHA. $s(\theta)$ and $n(\theta)$ can be calculated from $\mathrm{R}(\theta), \mathrm{R}\left(\theta+1^{\circ}\right), \mathrm{v}(\theta)$, and $\mathrm{v}\left(\theta+1^{\circ}\right)$ by satisfying equations (4-6):

$$
\begin{gathered}
R^{\prime}=R\left(\theta+1^{\circ}\right) R^{T}(\theta) \\
v^{\prime}=v\left(\theta+1^{\circ}\right)-R^{\prime} v(\theta)
\end{gathered}
$$




$$
R^{\prime} w+v^{\prime}=w+\operatorname{tn}(\theta)+(1-\cos \varphi) n(\theta) *(n(\theta) *(w-s(\theta)))+\sin \varphi n(\theta) *
$$

$$
(w-s(\theta))
$$

where R' and v' are the rotational matrix and translation vector representing the patellar motion from $\theta$ to $\theta+1^{\circ}$ knee flexion. $t$ is the patellar translation along the FHA, and $\varphi$ is the patellar rotation angle around the FHA. Equation (6) holds for any vector of w.

\section{Configuration of coordinate system}

A coordinate system based on the femur was established to facilitate the normalisation and inter-subject comparison of FHA tracking (Fig. 2). First, the sulcus of the medial epicondyle (point M in Fig. 2) and the prominence of the lateral epicondyle (point L in Fig. 2) were selected to form the femoral TEA [13], defined as the $x$-axis, with the midpoint of the TEA as the origin (point $\mathrm{O}$ in Fig. 2) and the direction from medial to lateral as positive. Second, the y-axis was established as the line passing through the origin and perpendicular to the TEA and the femoral shaft axis, with the direction from posterior to anterior as positive. As shown in Fig. 2A, the femoral shaft axis was formed by two section centres of the femoral shaft. Due to the limitation of the imaging size, only part of the distal femur can be identified through MR images (the length of the distal femur was less than $200 \mathrm{~mm}$ ). Therefore, shaft sections, 0.7 - and 0.8 - times the TEA length away from the origin, were automatically extracted to form the femoral shaft axis. Finally, the $\mathrm{z}$-axis was perpendicular to the $\mathrm{x}$-axis and $\mathrm{y}$-axis through the origin, and the direction from distal to proximal was positive.

To evaluate the influence of TEA determination on the subsequent calculation of 
patellar FHA parameters, the intra- and inter-rater intraclass correlation coefficients (ICCs) of these parameters were computed with the assistance of two clinical orthopedic doctors who were repeatedly invited to determine the TEAs on the 18 knee MR images with an interval time of two weeks or more.

\section{Parameters of patellar FHA}

To analyse the characteristics of FHA tracking, six parameters of location and orientation were quantified. (1) The intersection position (IP) between the FHA and midsagittal plane $\left(\mathrm{IP}_{\mathrm{y}}=\mathrm{y}\right.$ coordinate of $\mathrm{IP}, \mathrm{IP}_{\mathrm{z}}=\mathrm{z}$ coordinate of IP); (2) patellar rotational radius (PRR): the distance between the patellar centroid and its FHA [3]; (3) spatial angles between the FHA and femoral TEA $\left(\mathrm{A}_{\mathrm{F}-\mathrm{T}}\right)$; (4) angles between the FHA and coronal plane $\left(\mathrm{A}_{\mathrm{F}-\mathrm{C}}\right)$ : it was set positive when the FHA was located from posteromedial to anterolateral (Fig. 3A); (5) angles between the FHA and level plane $\left(A_{F-L}\right)$ : it was set positive when the FHA was located from superolateral to inferomedial (Fig. 3B). All parameters of the FHA location were normalised with the TEA length.

\section{Results}

The patellar FHA curves with knee flexion are shown in Additional file 2, and the 3D tracking of average patellar FHA is depicted in Fig. 4. Intra- and inter-rater ICCs of all aforementioned parameters of the FHA exceeded 0.95 (Table 2). The average length of femoral TEA was $7.85 \pm 0.53 \mathrm{~cm}$ (mean \pm standard deviation).

Table 2 Intra- and inter-rater reliability of patellar FHA parameters 


\begin{tabular}{|c|c|c|c|c|}
\hline & \multicolumn{2}{|c|}{ Intra-Rater } & \multicolumn{2}{|c|}{ Inter-Rater } \\
\hline & ICC & $(95 \% \mathrm{CI})$ & ICC & $(95 \% \mathrm{CI})$ \\
\hline $\mathrm{IP}_{\mathrm{y}}$ & 0.997 & (0.997 to 0.997$)$ & 0.995 & (0.994 to 0.995$)$ \\
\hline $\mathrm{IP}_{\mathrm{z}}$ & 0.989 & $(0.988$ to 0.990$)$ & 0.941 & (0.936 to 0.946$)$ \\
\hline PRR & 0.997 & (0.997 to 0.997$)$ & 0.996 & (0.996 to 0.997$)$ \\
\hline $\mathrm{A}_{\mathrm{F}-\mathrm{T}}$ & 0.981 & (0.980 to 0.983$)$ & 0.964 & (0.960 to 0.967$)$ \\
\hline $\mathrm{A}_{\mathrm{F}-\mathrm{C}}$ & 0.979 & $(0.978$ to 0.981$)$ & 0.965 & (0.962 to 0.968$)$ \\
\hline$A_{F-L}$ & 0.990 & $(0.989$ to 0.991$)$ & 0.983 & (0.981 to 0.984$)$ \\
\hline
\end{tabular}

\section{Tracking of patellar FHA}

With the knee flexing, the patellar FHA moved forward after shifting upward from the posterosuperior position about TEA. Specifically, the average IP moved backward and upward from the position of 0.1 behind and 0.5 below the TEA, reaching positions of and upward of the TEA, reaching 0.25 right above the TEA at $60^{\circ}$ flexion. During $60^{\circ}$ $90^{\circ}$ knee flexion, the average IP continued to move forward to the position of 0.1 in front of the TEA (Fig. 5A-B, Fig. 6). During $0^{\circ}-90^{\circ}$ knee flexion, the trajectory of the average IP was roughly L-shaped (Fig. 6).

\section{PRR}

271 In the first $20^{\circ}$ of the early stage, the PRR fluctuated between 0.4 and 1.5 , and varied to the range of $0.4-0.9$ during $20^{\circ}-45^{\circ}$ flexion. In the middle stage, PRR fluctuated 
within the range of $0.45-0.9$. The average PRR increased during $0^{\circ}-10^{\circ}$ flexion and then gradually decreased (Fig. 5C). Regardless of the real-time change of PRR, the average PRR in the early and middle stages was $0.72 \pm 0.14$ times the TEA length $(5.65 \pm 1.09$ $\mathrm{cm})$.

\section{Orientation of patellar FHA}

In the first $20^{\circ}$ of the early stage, the $\mathrm{A}_{\mathrm{F}-\mathrm{T}}$ changed from $10^{\circ}-80^{\circ}$ to $0^{\circ}-30^{\circ}$. During $20^{\circ}$ $90^{\circ}$ knee flexion, the $\mathrm{A}_{\mathrm{F}-\mathrm{T}}$ of $16 / 18$ subjects fluctuated between $0^{\circ}$ and $20^{\circ}$, and the average $\mathrm{A}_{\mathrm{F}-\mathrm{T}}$ was maintained at approximately $10^{\circ}$ (Fig. 5D). In the late stage, $\mathrm{A}_{\mathrm{F}-\mathrm{T}}$ tended to increase as the knee flexed along with the increase of individual discrepancy. In the first $20^{\circ}$ of the early stage, the individual differences of $\mathrm{A}_{\mathrm{F}-\mathrm{C}}$ reached $80^{\circ}$, and the $A_{F-C}$ of $16 / 18$ subjects changed from $\left(-30^{\circ}\right)-20^{\circ}$ to $\left(-10^{\circ}\right)-10^{\circ}$. During $20^{\circ}-90^{\circ}$ knee flexion, the $A_{F-C}$ of every subject continued to fluctuate within the range of $-10^{\circ}-10^{\circ}$. In the late stage, the patellar FHA of four subjects deviated from the coronal plane, and the others roughly maintained the previous direction. The average $\mathrm{A}_{\mathrm{F}-\mathrm{C}}$ was less than $5^{\circ}$ in the early and middle stages, and less than $2^{\circ}$ during $20^{\circ}-90^{\circ}$ of knee flexion (Fig. $5 \mathrm{E})$.

In the early stage, the $A_{F-L}$ of $17 / 18$ subjects changed from $\left(-80^{\circ}\right)-20^{\circ}$ to $0^{\circ}-20^{\circ}$, and the average $A_{F-L}$ changed from $-25^{\circ}$ to $10^{\circ}$ (Fig. $5 \mathrm{~F}$ ). In the middle stage, this angle gradually changed to the range of $\left(-20^{\circ}\right)-0^{\circ}$, with an average $\mathrm{A}_{\mathrm{F}-\mathrm{L}}$ changing to about $10^{\circ}$, and the $\mathrm{A}_{\mathrm{F}-\mathrm{L}}$ in $13 / 18$ subjects equalled $0^{\circ}$ at $50^{\circ}-70^{\circ}$ knee flexion. In the late stage, the individual differences of $\mathrm{A}_{\mathrm{F}-\mathrm{L}}$ tended to be greater than that in the former stage. 


\section{Discussion}

297 Patellofemoral pain is a common and refractory disease; however, its pathogenesis is 298 debatable [14]. Considering that joint kinematics is the mechanistic link between 299 musculoskeletal anatomy and joint function, a complete understanding of the physical patellofemoral dynamics is critical in clinical practice and trial studies. FHA is an

301 essential functional property of a joint [15]; however, its real-time location and 302 orientation in the patellofemoral joint remains unknown. With the leverage of the static 303 MR methodology, we calculated the continuous patellar FHA during knee flexion and found that patellar FHA was not fixed, exhibiting a close relationship with femoral TEA 305 in both location and orientation. quantitatively demonstrated in vivo with a relatively large normal population. All the intra- and inter-rater ICCs of the FHA parameters above were greater than 0.95 (Table 2), indicating an excellent repeatability of the TEA determination and the establishment of its coordinate system which has minor impact on the description of location and orientation of FHA. Our previous study confirmed the reliability of the static MR methodology in calculating the patellar trajectory and FHA [11]. Moreover, the current study was an in vivo study involving all soft tissues, which was closer to the physiological state than the two cadaver studies involving patellar FHA available to date $[3,9]$. The 3D average tracking of FHA and its intersections with medial, central, and lateral sagittal planes were presented, and six meaningful parameters regarding the 
location and orientation of the patellar FHA were obtained. These parameters exhibited different changes in the early, middle, and late stages.

The variation range and standard deviation of the FHA varied with knee flexion angles. In the early stage of knee flexion, especially the stage before $20^{\circ}$ flexion, the variation range of all six parameters tended to be larger than that in the middle stage, and with a larger standard deviation among subjects. This might be because the patella had not been captured by the trochlear groove and was only regulated by the soft tissue, which made the patella unstable and most patella dislocation and subluxation occurred in this stage [16]. The variation range and standard deviation of the patellar FHA became smaller in the middle stage, resulting from the enhancement of femoral condyles and soft tissues on patellar movement after entering the trochlear groove [16]. During $20^{\circ}-90^{\circ}$ flexion, the osseous restriction of the trochlear groove guided the patella to follow a uniplanar and circular path [3]. In the late stage, however, the fluctuation of the FHA increased again. This was likely to attribute to the transformation that the patella might move away from the circular pathway in deep flexion when it moves onto the bilateral femoral condyles, as Iranpour et al. speculated [3]. In addition, a higher percentage error beyond $90^{\circ}$ flexion was observed in the tibiofemoral kinematics [13], verifying that knee kinematics in deep flexion could no longer be represented as a rotation around a relatively fixed axis. Therefore, designing a longer and wider proximal femoral component would be conducive to capturing the patella especially for those with trochlear dysplasia, apart from that the enhancement of the restriction of the trochlear groove could ensure patellofemoral stability in the middle 
stage. To restore the standard FHA tracking in the late stage, proper transition between the femoral condyles and curvature of the trochlear groove is essential.

The current study indicated that patellar FHA was not fixed during knee flexion, presenting an overall L-shaped tracking in the midsagittal plane of the femur. Specifically, the average patellar FHA moved to the position of $0.1(7.9 \mathrm{~mm})$ anterior and $0.25(19.6 \mathrm{~mm})$ above the TEA from the position of $0.1(7.9 \mathrm{~mm})$ posterior and $0.05(4.0 \mathrm{~mm})$ below the TEA in the early and middle stages. However, Coughlin et al. [9] calculated that the origin of the patellar tracking arc was $9.6 \mathrm{~mm}(7.0-12.5 \mathrm{~mm})$ anterior and $11.6 \mathrm{~mm}(8.3-15.6 \mathrm{~mm})$ proximal to the TEA on average, without adequate attention to the real-time nature of its changes. The FHA translation was mainly caused by the diminishing radius of the trochlear groove curvature [17]. The decrease of patellar rotational radius during knee flexion increased additional superior motion of the patella, which caused the FHA shift anteriorly. This phenomenon is similar to the motion of tibiofemoral FHA previously reported by Sheehan et al. [8], who exemplified that an extra $1.1 \mathrm{~mm}$ of superior motion of the tibia during extension would result in a $25.0 \mathrm{~mm}$ posterior displacement of the tibiofemoral FHA. In addition, the tibial plateau moved anteriorly relative to the femoral condyles during knee flexion and was more significant especially beyond $45^{\circ}$ flexion, called rollback of the femur $[15,18]$. Meanwhile, the patella might display an additional anterior shift with traction from the patellar tendon, resulting in the inferior displacement of the FHA (Fig. 5B).

In the early and middle stages, the PRR fluctuated between 0.6 and 0.8 times the TEA length, averaging $56.5 \pm 10.9 \mathrm{~mm}$, larger than that in previous studies (26.4-46.9 
$\mathrm{mm})[3,9]$. This is due to the different methods studied. For example, Coughlin et al.

[9] chose the most prominent point on the dorsal ridge of the patella to fit the arc motion, differing from the patellar geometric center selected in our study. From $20^{\circ}$ of knee flexion when the patella was captured by the trochlear groove, the average rotational radius showed a slight decrease, which was related to the slight diminishing radius of curvature of the trochlear groove [17]. Thus, to normalise the PRR, it is important to restore the trochlear groove's geometry when designing prostheses and selecting appropriate sizes for total knee arthroplasty. If an oversized femoral component with an increased radius of the trochlear groove curvature was installed, the range of motion would be restricted [19]. By contrast, extension lag and weakness would occur if the femoral component was undersized due to the smaller moment arm of the patellofemoral joint.

In this study, the orientation of the patellar FHA was predominantly in a mediolateral direction, with an average angle between the FHA and TEA not exceeding $10^{\circ}$ during most phages of knee flexion. However, at the initial stage of flexion, there were larger angles and deviations between the FHA and TEA, level plane, and coronal plane, which were caused by the fact that the patella did not follow a circular path [3]. From full extension, the patella shifted medially with the guidance of the medial retinaculum [20], so that its FHA seemed to be oblique relative to the TEA, with the FHA orientation from superomedial to inferolateral. That is, medial shift of the patella increased $A_{F-T}$ and $\mathrm{A}_{\mathrm{F}-\mathrm{L}}$. As the patellar shift slowed down, both $\mathrm{A}_{\mathrm{F}-\mathrm{T}}$ and $\mathrm{A}_{\mathrm{F}-\mathrm{L}}$ tended to decrease as well. Beyond $20^{\circ}$ knee flexion, the patella shifted laterally in the trochlear groove [20, 
21], and made the FHA orientation posterolateral-anteromedial. In the middle stage, $\mathrm{A}_{\mathrm{F}-}$ L tended to decrease again with the patellar shift slowing down. As the knee flexed, however, the effect of patellar shift on $A_{F-L}$ gradually reduced, while that on $A_{F-C}$ increased. In the late stage, the $\mathrm{A}_{\mathrm{F}-\mathrm{C}}$ transferred to be posterolateral-anteromedial, possibly due to the slight medial shift of the patella [22].

To facilitate clinical application, we depicted the relationship between the FHA and femoral TEA. Because the TEA is an anatomic marker which is easy to identify and widely used clinically, and the present results showed that the patellar FHA was close to the TEA, it would be reliable to assess the FHA with the TEA as a reference. The insertion point of the medial and lateral retinaculum of the patellofemoral joint was near the sulcus of the medial epicondyle and the prominence of the lateral epicondyle, respectively [16], which was speculated as one of the causes of why the FHA was close to the TEA. To date, the TEA has been regarded as an essential reference when installing the trochlear prosthesis in patellofemoral arthroplasty, and relatively successful clinical results have been achieved [23], which might be related to the close relationship between the patellar FHA and TEA. Nevertheless, the FHA was nonoverlapping with TEA even if in the middle stage. This could be explained by the fact that the patella did not follow a complete circle that is influenced by the morphology of the trochlear groove, which has resulted in the ever-changing FHA with knee flexion.

There were some limitations to this study. First, the patellar FHA was obtained under non-weight-bearing conditions without muscle loads, which might differ from that under weightbearing conditions. Therefore, further studies about the effect of loading 
on the FHA are necessary. Second, the patellar motion calculated from finite static MR images was not completely congruent with the realistic one, but it was helpful for us to understand the spatial distribution of the patellar FHA, and additionally the accuracy of this method has been confirmed in our previous study [10]. Third, due to the limited space of the MR machine, the maximum knee flexion angles of half of the 18 subjects were less than $110^{\circ}$, which could be improved by further equipment updates.

\section{Conclusions}

The patellar FHA was not fixed with knee flexion, showing a close relationship with the femoral TEA in both location and orientation. The tracking of the intersections between the FHA and midsagittal plane was roughly "L-shaped", moving from the posteroinferior aspect of the TEA to the anterosuperior of TEA. During $20^{\circ}-90^{\circ}$ knee flexion, the angle between the FHA and TEA was approximately $10^{\circ}$ and the FHA was approximately parallel to the level plane. The results may help us better understand the motion of the patellofemoral joint and further deal with troublesome patellofemoral disorders.

\section{List of abbreviations}

patellar finite helical axis (FHA)

trans-epicondylar axis (TEA)

magnetic resonance (MR)

three-dimensional (3D) 
intraclass correlation coefficient (ICC)

the intersection position between FHA and midsagittal plane (IP)

the intersection position between FHA and medial sagittal plane (IP $\left.{ }_{\mathrm{M}}\right)$

the intersection position between FHA and lateral sagittal plane $\left(\mathrm{IP}_{\mathrm{L}}\right)$

y coordinate of IP $\left(\mathrm{IP}_{\mathrm{y}}\right)$

$\mathrm{z}$ coordinate of IP $\left(\mathrm{IP}_{\mathrm{z}}\right)$

patellar rotational radius $(\mathrm{PRR})$

angles between patellar FHA and TEA (AF-T)

angles between patellar FHA and coronal plane $\left(\mathrm{A}_{\mathrm{F}-\mathrm{C}}\right)$

angles between patellar FHA and level plane $\left(\mathrm{A}_{\mathrm{F}-\mathrm{L}}\right)$

\section{Reference}

[1]. Esfandiarpour, F., et al., In-vivo patellar tracking in individuals with patellofemoral pain and healthy individuals. Journal of Orthopaedic Research: Official Publication of the Orthopaedic Research Society, 2018.

[2]. Jin, J. and E. Jones, Patellofemoral pain. JAMA, 2018. 319(4): p. 418.

[3]. Iranpour, F., et al., Patellofemoral joint kinematics: The circular path of the patella around the trochlear axis. Journal of Orthopaedic Research: Official Publication of the Orthopaedic Research Society, 2010. 28(5): p. 589-594.

[4]. Kedgley, A.E., E.J. McWalter and D.R. Wilson, The effect of coordinate system variation on in vivo patellofemoral kinematic measures. Knee, 2015. 22(2): p. 88-94. 
[5]. Yu, Z., et al., Research methods and progress of patellofemoral joint kinematics: A review. Journal of Healthcare Engineering, 2019. 2019: p. 9159267.

[6]. Konda, S., et al., Comparison of finite helical axes of normal and anatomically designed prosthetic knees. Clinical Biomechanics (Bristol, Avon), 2019. 65: p. 57-64. [7]. Wade, F.E., L.J. Hickox and S.J. Piazza, Achilles tendon moment arms are similar when computed using a single fixed axis versus a moving instantaneous helical axis. Journal of Biomechanics, 2020. 109: p. 109907.

[8]. Sheehan, F.T., The finite helical axis of the knee joint (a non-invasive in vivo study using fast-PC MRI). Journal of Biomechanics, 2007. 40(5): p. 1038-1047.

[9]. Coughlin, K.M., et al., Tibial axis and patellar position relative to the femoral epicondylar axis during squatting. Journal of Arthroplasty, 2003. 18(8): p. 1048-1055. [10]. Yao, J., et al., Patella tracking calculation from patellofemoral positions at finite angles of knee flexion. Medical Engineering \& Physics, 2018. 62: p. 1-6.

[11]. Yao, J., et al., In vivo measurements of patellar tracking and finite helical axis using a static magnetic resonance based methodology. Medical Engineering \& Physics, 2014. 36(12): p. 1611-1617.

[12]. Spoor, C.W. and F.E. Veldpaus, Rigid body motion calculated from spatial coordinates of markers. Journal of Biomechanics, 1980. 13(4): p. 391-393.

[13]. Asano, T., M. Akagi and T. Nakamura, The functional flexion-extension axis of the knee corresponds to the surgical epicondylar axis - In vivo analysis using a biplanar image-matching technique. Journal of Arthroplasty, 2005. 20(8): p. 1060-1067.

[14]. Lankhorst, N.E., S.M. Bierma-Zeinstra and M. van Middelkoop, Risk factors for 
patellofemoral pain syndrome: A systematic review. Journal of Orthopaedic \& Sports Physical Therapy, 2012. 42(2): p. 81-94.

[15]. van den Bogert, A.J., C. Reinschmidt and A. Lundberg, Helical axes of skeletal knee joint motion during running. Journal of Biomechanics, 2008. 41(8): p. 1632-1638. [16]. Greiwe, R.M., et al., Anatomy and biomechanics of patellar instability. Operative Techniques in Sports Medicine, 2010. 18(2): p. 62-67.

[17]. Du, Z., et al., Do size, shape, and alignment parameters of the femoral condyle affect the trochlear groove tracking? A morphometric study based on 3D-computed tomography models in Chinese people. BMC Musculoskeletal Disorders, 2017. 18(1): 4.

[18]. Feng, Y., et al., In-vivo analysis of flexion axes of the knee: Femoral condylar motion during dynamic knee flexion. Clinical Biomechanics, 2016. 32: p. 102-107.

[19]. Lo, C.S., S.J. Wang and S.S. Wu, Knee stiffness on extension caused by an oversized femoral component after total knee arthroplasty-A report of two cases and a review of the literature. Journal of Arthroplasty, 2003. 18(6): p. 804-808.

[20]. Amis, A.A., W. Senavongse and A.M. Bull, Patellofemoral kinematics during knee flexion-extension: An in vitro study. Journal of Orthopaedic Research: Official Publication of the Orthopaedic Research Society, 2006. 24(12): p. 2201-2211.

[21]. Merican, A.M. and A.A. Amis, Iliotibial band tension affects patellofemoral and tibiofemoral kinematics. Journal of Biomechanics, 2009. 42(10): p. 1539-1546.

[22]. Nha, K.W., et al., In vivo patellar tracking: Clinical motions and patellofemoral indices. Journal of Orthopaedic Research: Official Publication of the Orthopaedic 
Research Society, 2008. 26(8): p. 1067-1074.

494 [23]. Remy, F., Surgical technique in patellofemoral arthroplasty. Orthopaedics \& 495 Traumatology, Surgery \& Research, 2019. 105(1S): p. S165-S176.

496

497

498

499

500

501

502

503

504

505

506

507

508

509

510

511

512

513

514 
Fig. 1 Geometry reconstruction of the knee joint. (A) The knee joint is scanned with the MR machine at full extension and at $30^{\circ}, 60^{\circ}, 90^{\circ}$, and maximum angle of knee flexion. (B) Based on the MR images, the 3D models of the femur, patella, and tibia are developed with the medical image processing software, Mimics. (C) Patellar tracking is calculated from the above knee models at five angles of knee flexion. medial epicondyle (point $\mathrm{M}$ ) and the highest point of the lateral epicondyle (point L) are selected to form TEA, which is defined as the x-axis, with the midpoint of TEA as

Fig. 3 Definition of the orientation of the patellar FHA. (A) With respect to the angles between the patellar FHA and coronal plane $\left(\mathrm{A}_{\mathrm{F}-\mathrm{C}}\right)$, it is set positive when the FHA is located from posteromedial to anterolateral (line $\alpha$ ) and negative when the FHA is 
located from posterolateral to anteromedial (line $\beta$ ). (B) With respect to the angles between the FHA and level plane ( $\left.\mathrm{A}_{\mathrm{F}-\mathrm{L}}\right)$, it is set positive when FHA is located from superolateral to inferomedial (line $\gamma$ ) and negative when the FHA is located from superomedial to inferolateral (line $\delta$ ).

Fig. 4 Three-dimensional tracking of the average patellar FHA. The average patellar FHA is continuously changed with knee flexion, with a gradient of colour from purple to red.

Fig. 5 Average FHA parameters during knee flexion. (A, B) The average IP moves backward and upward from the position of 0.1 behind and 0.5 below the TEA, reaching the position of 0.2 posterior and 0.05 above the TEA at $10^{\circ}$ knee flexion; subsequently, it turnes to move forward and upward of the TEA, reaching 0.25 right above the TEA at $60^{\circ}$ flexion. During $60^{\circ}-90^{\circ}$ knee flexion, the average IP continues to move forward to the position of 0.1 front the TEA. (C) The average PRR increases during $0^{\circ}-10^{\circ}$ flexion and then gradually decreases. (D) During $20^{\circ}-90^{\circ}$ knee flexion, average AF-T maintains at about $10^{\circ}$. (E) The average $A_{F-C}$ is less than $5^{\circ}$ in early and middle stages and is less than $2^{\circ}$ during $20^{\circ}-90^{\circ}$ knee flexion. (F) In the early stage, the average $A_{F-L}$ changes from $-25^{\circ}$ to $10^{\circ}$; and in the middle stage, the average $A_{F-L}$ changes to about $10^{\circ}$

Fig. 6 The intersection position between the FHA and midsagittal plane. During $0^{\circ}-90^{\circ}$ 
559 knee flexion, the trajectory of the average intersection position is roughly L-shaped.

560

561

$562 \quad$ Additional files

563 The continuous motion process of the patella and patellar FHA is shown in the form of

564 animation (Additional file 1). The curves of the six parameters of the FHA with knee

565 flexion are displayed in Additional file 2. 
Figures
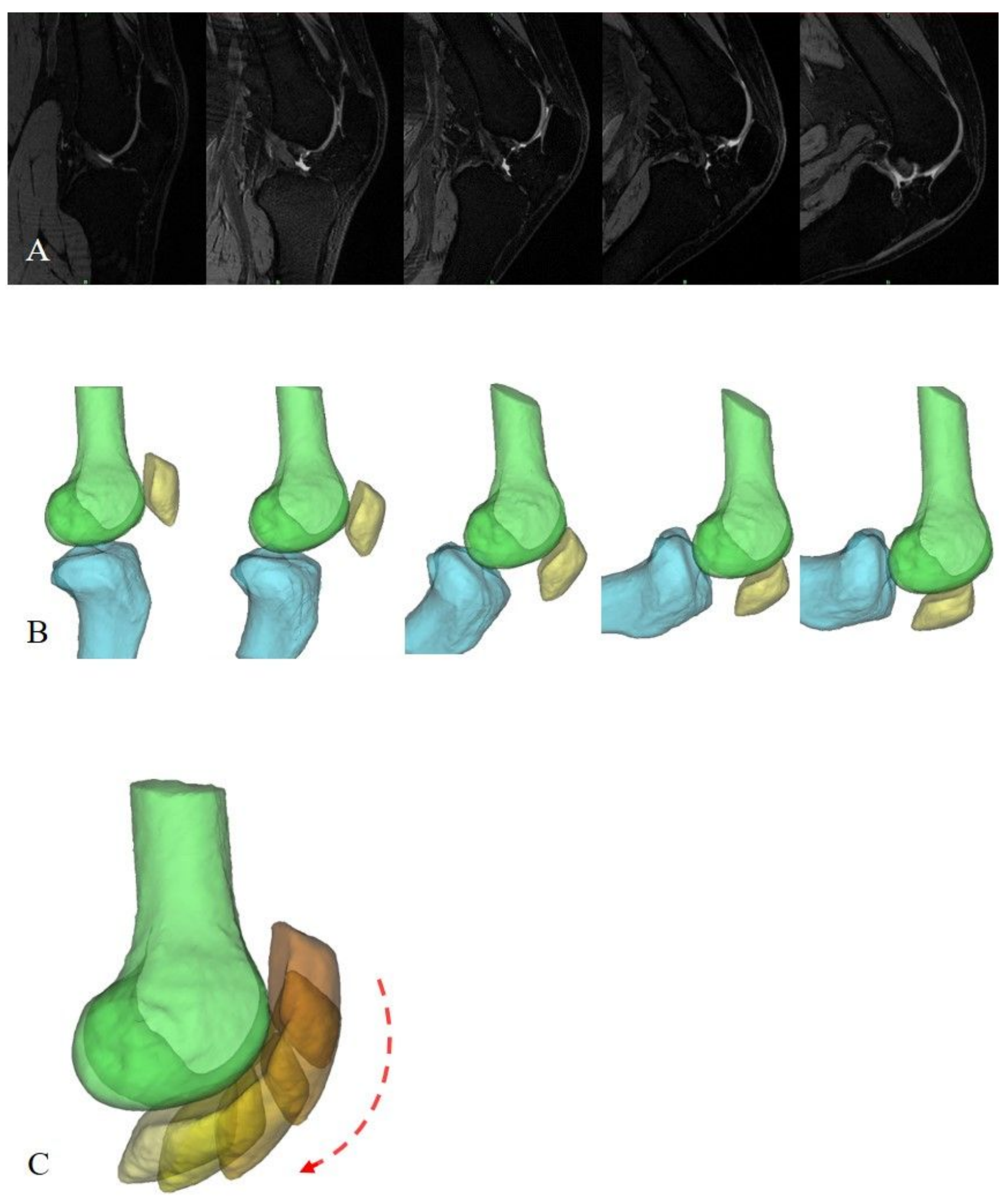

\section{Figure 1}

Geometry reconstruction of the knee joint. (A) The knee joint is scanned with the MR machine at full extension and at $30^{\circ}, 60^{\circ}, 90^{\circ}$, and maximum angle of knee flexion. (B) Based on the MR images, the 3D 
models of the femur, patella, and tibia are developed with the medical image processing software, Mimics. (C) Patellar tracking is calculated from the above knee models at five angles of knee flexion.
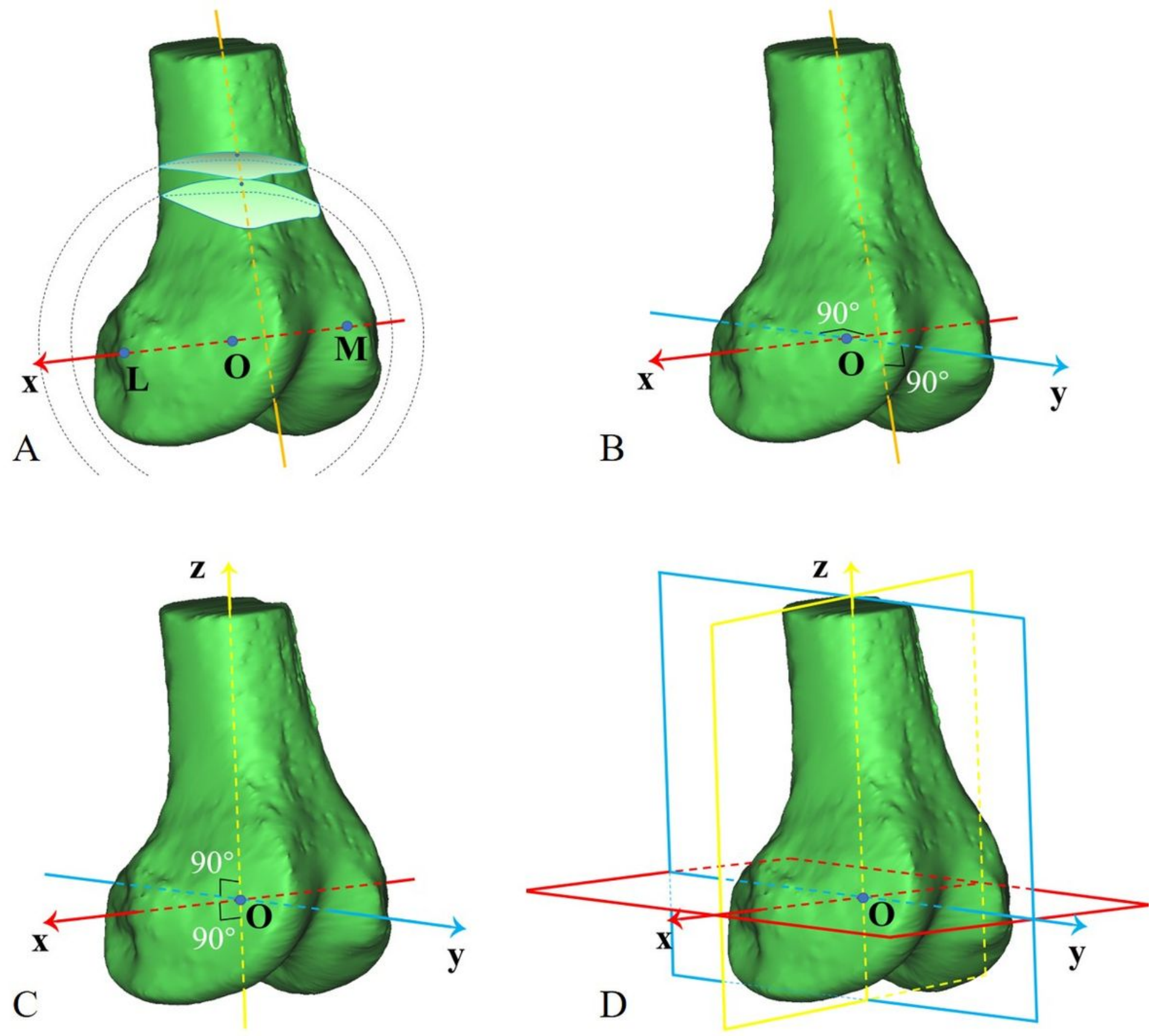

Figure 2

A coordinate system is established based on the femur. (A) The fovea of the medial epicondyle (point M) and the highest point of the lateral epicondyle (point $L$ ) are selected to form TEA, which is defined as the $x$-axis, with the midpoint of TEA as the origin (point 0 ); two virtual balls-with the origin as their centres and with 0.7- and 0.8- times of the TEA length as their radius respectively-are crossed with the femur to obtain two section surfaces, whose centroids are linked to determine the femoral shaft axis. (B) The yaxis is defined as the line passing through the origin and perpendicular to the TEA and the femoral shaft 
axis meanwhile. (C) The $z$-axis is perpendicular to the $x$-axis and $y$-axis through the origin. (D) Three planes are determined by $x-, y$ - and $z$-axis (red: level plane; yellow: coronal plane; blue: midsagittal plane).
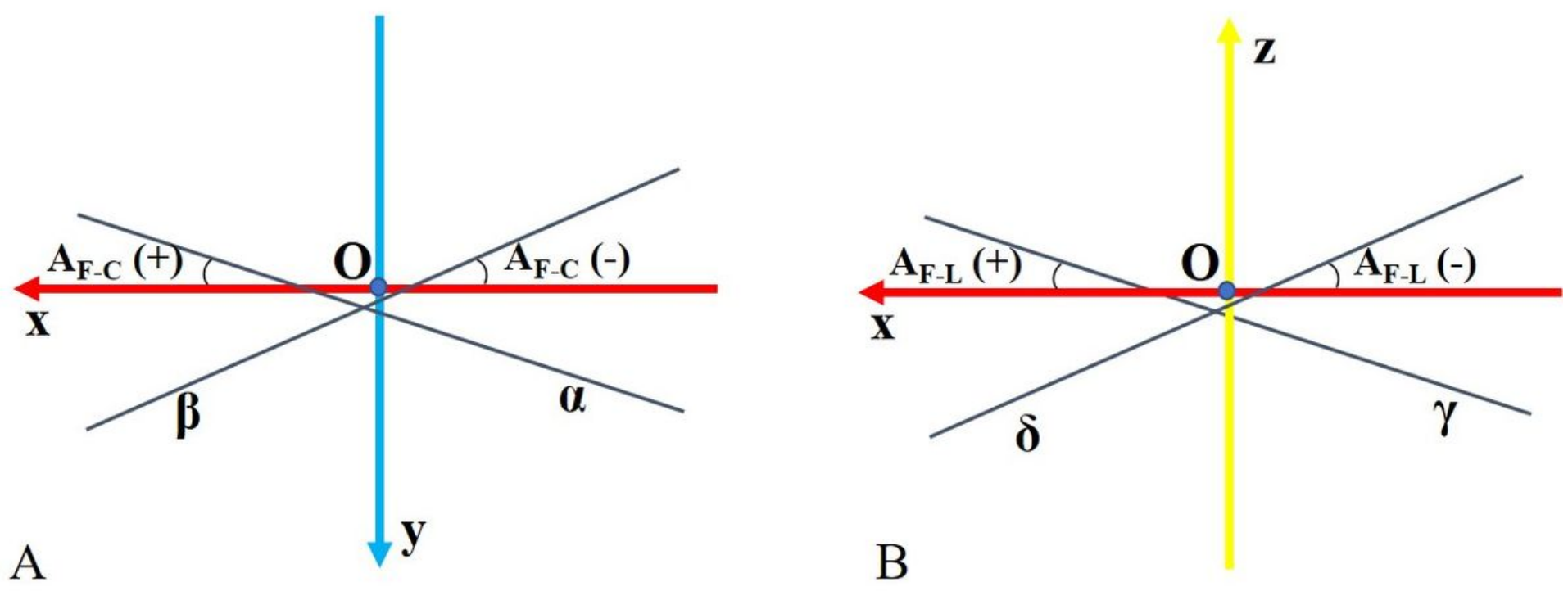

Figure 3

Definition of the orientation of the patellar FHA. (A) With respect to the angles between the patellar FHA and coronal plane (AF-C), it is set positive when the FHA is located from posteromedial to anterolateral (line $a$ ) and negative when the FHA is located from posterolateral to anteromedial (line $\beta$ ). (B) With respect to the angles between the FHA and level plane (AF-L), it is set positive when FHA is located from superolateral to inferomedial (line $\gamma$ ) and negative when the FHA is located from superomedial to inferolateral (line $\delta$ ). 

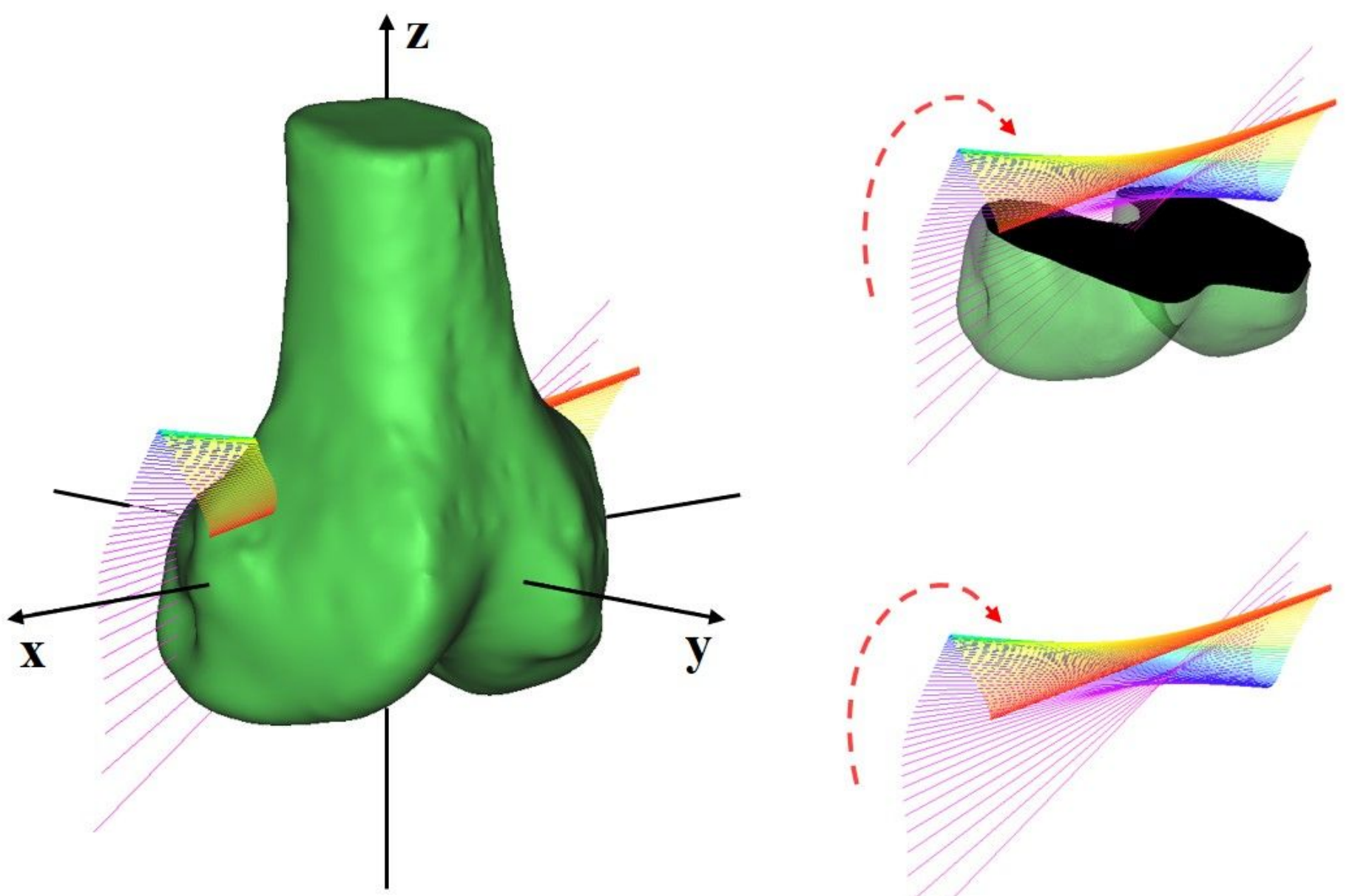

Figure 4

Three-dimensional tracking of the average patellar FHA. The average patellar FHA is continuously changed with knee flexion, with a gradient of colour from purple to red. 

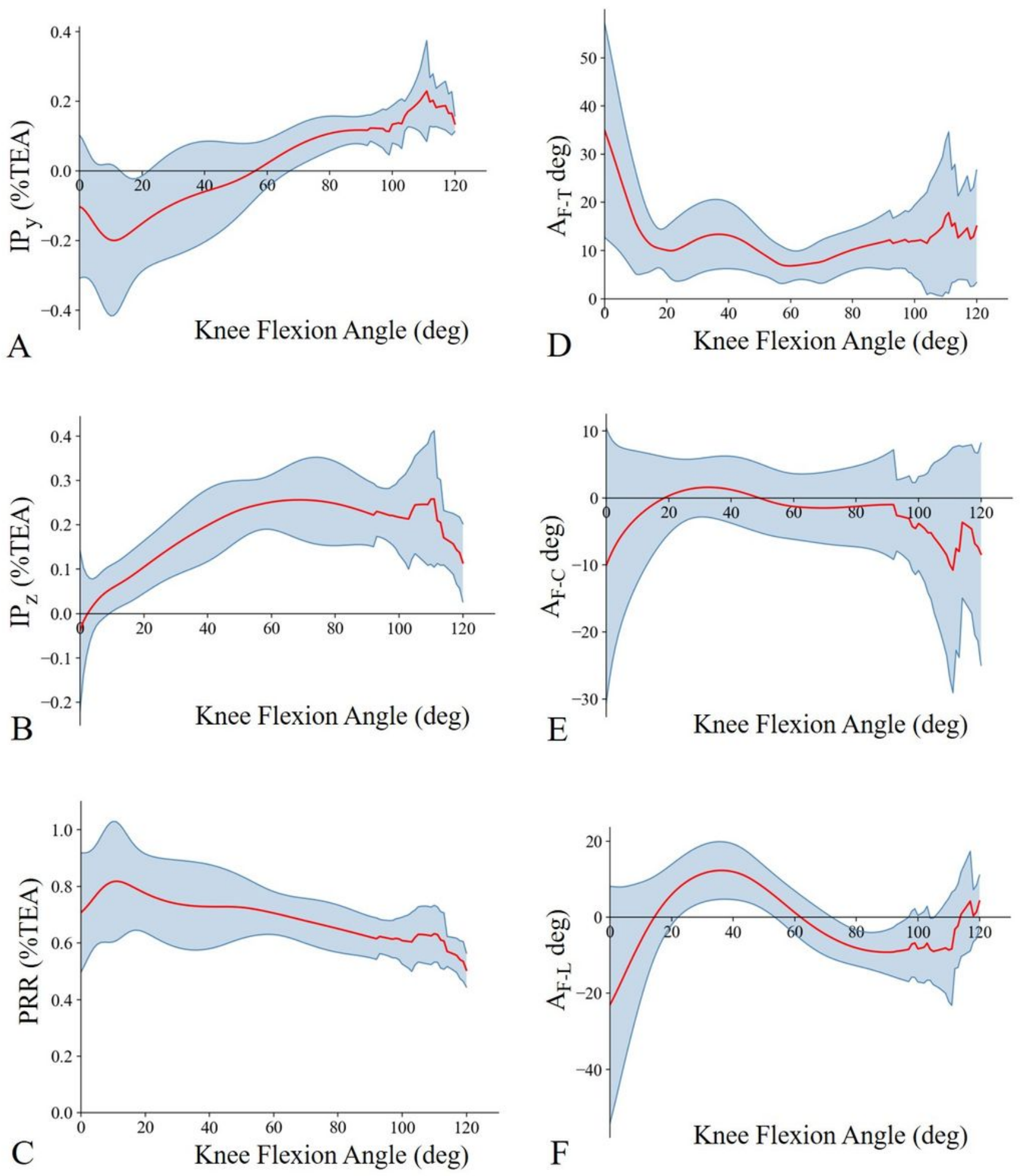

\section{Figure 5}

Average FHA parameters during knee flexion. (A, B) The average IP moves backward and upward from the position of 0.1 behind and 0.5 below the TEA, reaching the position of 0.2 posterior and 0.05 above the TEA at $10^{\circ}$ knee flexion; subsequently, it turnes to move forward and upward of the TEA, reaching 0.25 right above the TEA at $60^{\circ}$ flexion. During $60^{\circ}-90^{\circ}$ knee flexion, the average IP continues to move forward to the position of 0.1 front the TEA. (C) The average PRR increases during $0^{\circ}-10^{\circ}$ flexion and then 
gradually decreases. (D) During $20^{\circ}-90^{\circ}$ knee flexion, average AF-T maintains at about $10^{\circ}$. (E) The average AF-C is less than $5^{\circ}$ in early and middle stages and is less than $2^{\circ}$ during $20^{\circ}-90^{\circ}$ knee flexion. (F) In the early stage, the average AF-L changes from $-25^{\circ}$ to $10^{\circ}$; and in the middle stage, the average AF-L changes to about $-10^{\circ}$.

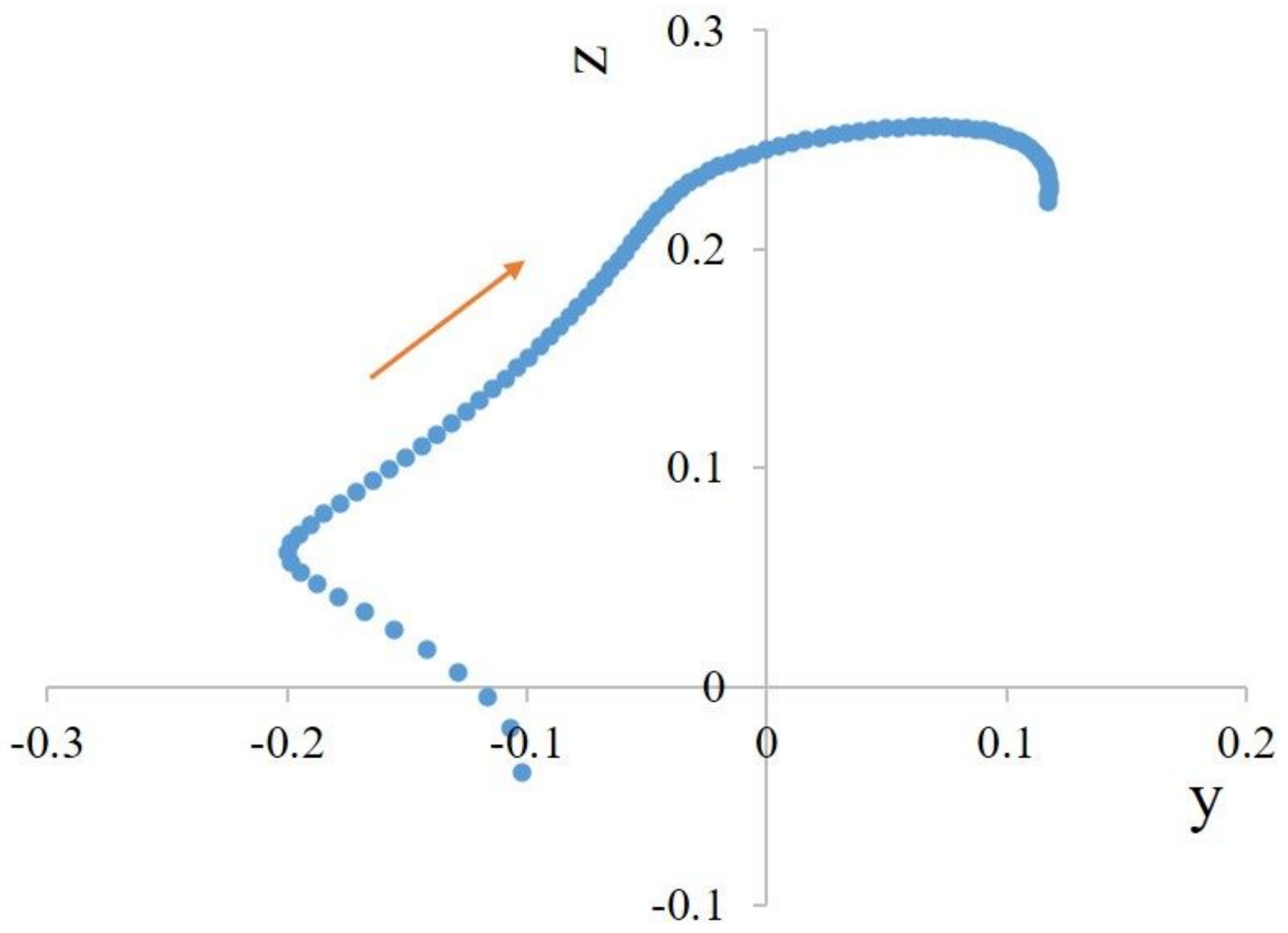

\section{Figure 6}

The intersection position between the FHA and midsagittal plane. During $0^{\circ}-90^{\circ}$ knee flexion, the trajectory of the average intersection position is roughly L-shaped.

\section{Supplementary Files}

This is a list of supplementary files associated with this preprint. Click to download.

- Additionalfile1.mp4

- Additionalfile2.jpg 